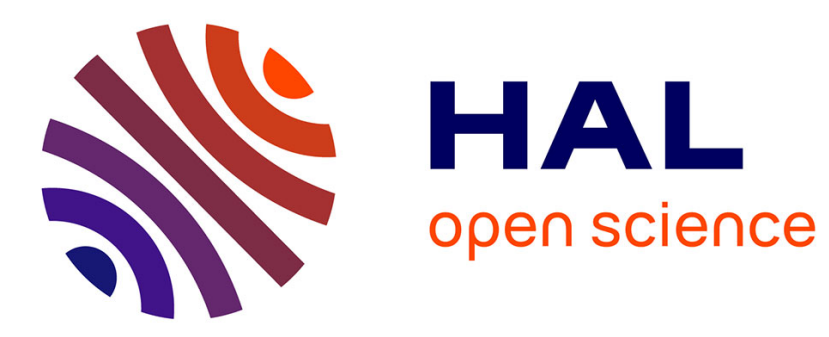

\title{
A Discontinuous Galerkin Variational Multiscale Approach to LES of Turbulent Flows
}

\author{
Marta de La Llave Plata, Eric Lamballais, Vincent Couaillier
}

\section{To cite this version:}

Marta de La Llave Plata, Eric Lamballais, Vincent Couaillier. A Discontinuous Galerkin Variational Multiscale Approach to LES of Turbulent Flows. Direct and Large-Eddy Simulation XI, , pp.47-52, 2019, 978-3-030-04915-7. 10.1007/978-3-030-04915-7_7 . hal-02859999

\section{HAL Id: hal-02859999 https://hal.science/hal-02859999}

Submitted on 8 Jun 2020

HAL is a multi-disciplinary open access archive for the deposit and dissemination of scientific research documents, whether they are published or not. The documents may come from teaching and research institutions in France or abroad, or from public or private research centers.
L'archive ouverte pluridisciplinaire HAL, est destinée au dépôt et à la diffusion de documents scientifiques de niveau recherche, publiés ou non, émanant des établissements d'enseignement et de recherche français ou étrangers, des laboratoires publics ou privés. 


\title{
A discontinuous Galerkin variational multiscale approach to LES of turbulent flows
}

\author{
Marta de la Llave Plata, Eric Lamballais, and Vincent Couaillier
}

\begin{abstract}
Three approaches to LES based on the DG method, namely, the nomodel, the standard LES and the VMS approaches, are compared for two lowMach-number configurations at high Reynolds number. The effect of the numerical flux function on the quality of the DG-LES solutions is also studied in the context of very coarse discretisations.
\end{abstract}

\section{Introduction and numerical method}

In recent work [1] we have developed a variational multiscale simulation (VMS) approach based on a modal discontinuous Galerkin (DG) method. The separation of scales is achieved in each element via projection onto the discontinuous modal space. In [1], the DG-VMS technique was applied to the Taylor-Green vortex (TGV) flow at $R e=3000$ demonstrating the potential of this approach to perform LES.

In this paper, we investigate the behaviour of the DG-VMS technique for two higher-Reynolds-number configurations, the TGV at $R e=20000$ and the flow past a circular cylinder at $R e=3900$ and 20000, which constitutes a more complex configuration, involving real boundary conditions and flow separation. The no-model as well as the standard LES approaches are also considered and confronted to DGVMS. For our model-based LES simulations two methods are employed: standard LES based on either the Smagorinsky model, referred to as DG-Smag, or the WALE model, denoted by DG-Wale, and the DG-VMS approach in combination with a

Marta de la Llave Plata

ONERA - FR-92322 Châtillon, France e-mail: marta.de_la_llave_plata@onera.fr

Eric Lamballais

Institute PPRIME, Université de Poitiers, France e-mail: eric.lamballais@univ-poitiers.fr

Vincent Couaillier

ONERA - FR-92322 Châtillon, France, e-mail: vincent.couaillier@onera.fr 
Smagorinsky eddy viscosity to model the effect of the unresolved scales on the small-scale resolved field. The results from the DG simulations are assessed by detailed comparisons against reference data from DNS and experiment. The effect of the numerical flux function on the different terms involved in the kinetic energy (k.e.) balance equation is also studied in the case of the TGV configuration.

The simulation results presented here have been performed using the compressible DG solver Aghora developed at ONERA [1,2]. The DG discretization is based on a modal approach, and over-integration is performed for de-aliasing purposes. As regards the numerical flux used in the discretisation of the convective terms the Roe and local Lax-Friedrichs (LLF) fluxes are employed in this work. We also use a modified version of the LLF flux, hereafter called $\alpha$ LLF, in which a parameter $\alpha=0.1$ scales its upwind component, thus allowing us to reduce the numerical dissipation introduced by the scheme. The time integration is performed using an explicit third-order accurate Runge-Kutta method.

\section{Taylor-Green vortex problem at $R e=20000$}

In this section, LES simulations of the TGV at $R e=20000$ and $M=0.1$ are carried out and compared to filtered DNS data. The reference DNS data have been generated using a 6th-order FD code on a grid composed of $3458^{3}$ nodes [3]. These data are filtered following a procedure similar to the one described in [1].

A very coarse grid composed of $28^{3}$ hexahedral elements is used in the LES. The degree of the polynomial basis is set to $p=8$ which leads to 9th-order accuracy in space. The number of degrees of freedom (DOFs) in the LES is thus $252^{3}$, which corresponds to approximately $1 / 14^{3}$ of the number of DOFs of the reference DNS.

We monitor the quantities involved in the balance of the volume-averaged k.e. $E$. For a DNS, this energy balance simply is given by $-\frac{d E}{d t}=\varepsilon+\varepsilon_{c}$, in which $-\frac{d E}{d t}$ is the k.e. dissipation, $\varepsilon=2 \frac{\mu}{\rho_{0} V} \int_{V} \mathbf{S}: \mathbf{S} d V$ is the viscous dissipation, and $\varepsilon_{c}=-\frac{1}{\rho_{0} V} \int_{V} p \nabla \cdot \mathbf{u} d V$ is the dissipation due to compressibility effects. Here, $\mathbf{S}$ denotes the rate-of-strain tensor, $\mu$ the molecular viscosity, $\rho_{0}$ the volume-averaged density, and $p$ the static pressure.

In an LES, however, the energy balance involves an additional term, $\varepsilon_{s g s}$, which is called the subgrid-scale (SGS) dissipation, leading to $-\frac{d E}{d t}=\varepsilon_{l s}+\varepsilon_{s g s}+\varepsilon_{c}$. The term $\varepsilon_{l s}$ is computed using the full resolved LES field in the expression for $\varepsilon$ provided above, and is now called the large-scale (LS) dissipation. As regards $\varepsilon_{s g s}$, there does not exist, in the general case, an explicit expression for this term, and in a posteriori computations it is simply obtained as $\varepsilon_{s g s}=-\frac{d E}{d t}-\varepsilon_{l s}-\varepsilon_{c}$. If the Mach number is low, $\varepsilon_{c}$ is expected to be negligible. We will see however that, due to under-resolution and other numerical errors, this term can actually take on very important values.

Figure 1 compares the evolution of $\varepsilon_{l s}, \varepsilon_{s g s}$, and $\varepsilon_{c}$, respectively, for the three different approaches considered. The LLF flux is employed in these simulations. 

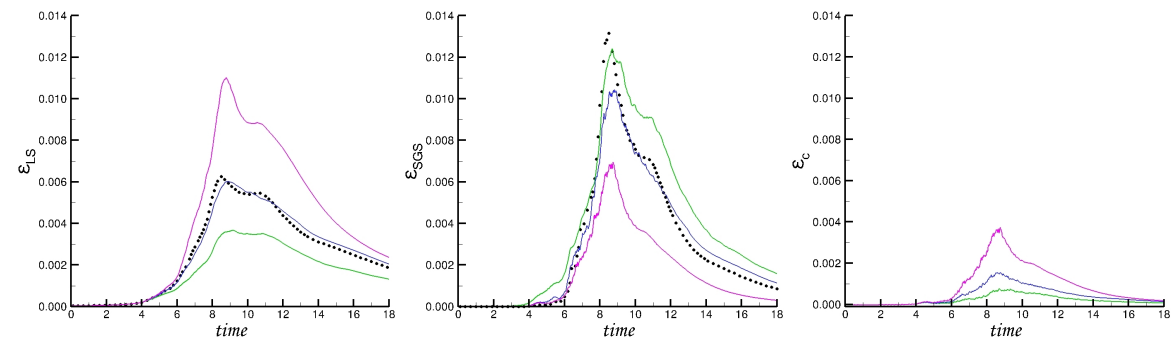

Fig. 1 From left to right, evolution of $\varepsilon_{l s}, \varepsilon_{s g s}$, and $\varepsilon_{c}$ for TGV at $R e=20000$. Filtered DNS: $\bullet$, no-model DG: -, DG-VMS: —, DG-Smag: -
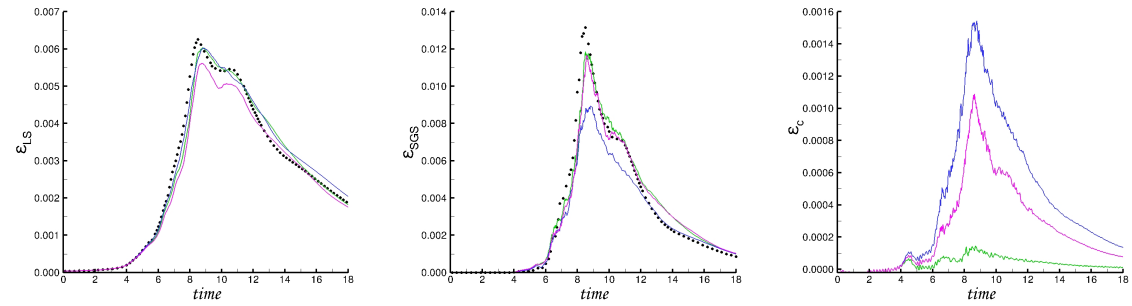

Fig. 2 From left to right, evolution of $\varepsilon_{l s}, \varepsilon_{s g s}$, and $\varepsilon_{c}$ for DG-VMS of the TGV at $R e=20000$. Filtered DNS: $\bullet$, LLF flux: —, $\alpha$ LLF flux: - , Roe flux: - .

It is straightforward to see from Fig. 1 that the no-model DG exhibits an underdissipative behaviour, with a peak of LS dissipation which is largely above that of the reference, and the lowest level of SGS dissipation, well below the reference. The opposite behaviour is observed for the DG-Smag simulation which presents the lowest level of LS dissipation. Overall, we see that the DG-VMS solution appears to provide the closest results to the reference with an evolution of LS dissipation almost on top of the reference. The evolution of SGS dissipation, in between the no-model DG and the DG-Smag solutions, is however far from the reference data. If we now examine the evolution of $\varepsilon_{c}$, we notice that, contrary to what we would expect, the dissipation due to pressure dilatation is far from being negligible. The levels of $\varepsilon_{c}$ tend to be reduced with the introduction of SGS viscosity, the DG-Smag simulation presenting the lowest values, while for the no-model DG $\varepsilon_{c}$ is even larger than $\varepsilon_{s g s}$ near the peak. Further investigation into the behaviour of $\varepsilon_{c}$ using different discretisations (not show here for brevity) results in the following observations: the levels of $\varepsilon_{c}$ are reduced when the number of DOFs is increased, when the polynomial order $p$ is increased (at equal number of DOFs), and when the SGS dissipation is increased. These observations suggest that, in the context of this low-Mach-number flow, a non-negligible value of $\varepsilon_{c}$ is clearly linked to under-resolution, and thus somehow to the jumps in the DG solution, which lead to excessive numerical dissipation via the flux upwinding term.

In a quest to get rid of these spurious compressibility effects we have studied the effect of the numerical flux on the solution. Recent research [4] has actually shown 
the strong influence that the numerical flux employed can have on the accuracy of no-model DG simulations of the Euler equations. In Fig. 2 we therefore compare three simulations using different fluxes: the standard LLF, the $\alpha \operatorname{LLF}$ (with $\alpha=0.1$ ), and the Roe flux. Only the DG-VMS approach is shown in this figure. The DGSmag approach yielded similar trends to the DG-VMS, while the no-model DG simulations crashed when the $\alpha$ LLF or the Roe flux were considered. By looking at the evolution of $\varepsilon_{c}$ shown in Fig. 2 we readily see that this term is linked to a numerical dissipation mainly introduced by the numerical flux at this low Mach number. Note that, for clarity, the y-axis scale in the plot of $\varepsilon_{c}$ has been stretched by a factor of about 10 with respect to the other graphs. The better performance displayed by the Roe flux with respect to the LLF scheme was pointed out in [4]. In terms of agreement of the three dissipation components $\varepsilon_{l s}, \varepsilon_{s g s}$, and $\varepsilon_{c}$ with the filtered DNS, the use of the $\alpha$ LLF flux is found here to provide the best results over the whole course of the simulation.

Finally, we would like to highlight the importance of examining each of the terms involved in the balance equation, rather than focusing on the analysis of an isolated quantity such as the k.e. dissipation, or even the enstrophy alone. Indeed, we have seen from this study that a successful LES simulation is one that is able to yield accurate results for $\varepsilon_{l s}, \varepsilon_{s g s}$, and $\varepsilon_{c}$, separately.

\section{Flow past a circular cylinder at $R e=3900$ and 20000}

In this section, we consider the flow past a circular cylinder at Reynolds numbers, $R e=3900$ and 20000 based on the cylinder diameter $D$, and the freestream velocity $U_{c}$. Periodicity of the flow is assumed in the spanwise direction, and an isothermal no-slip boundary condition is imposed on the cylinder wall. The freestream Mach number is set to $M=0.2$. A fourth-order hexahedral O-type grid composed of 20736 elements is considered in a computational domain with radial and spanwise extension of $25 D$ and $\pi D$, respectively. The mesh resolution at the wall is $\Delta r=0.05 D$. The polynomial degree is set to $p=4$, which leads to fifth-order accuracy in space, and an effective resolution at the wall of $\Delta r / p+1=0.01 D$. The number of DOFs of the problem is 2.59 million (Mdofs).

For the lowest Re, three different approaches are considered: no-model DG, DGWale, and DG-VMS. For $R e=20000$ only the DG-Smag and the DG-VMS approaches yielded stable simulations and their outcome is reported here.

Following the conclusions drawn from the previous section, the $\alpha$ LLF flux is used in the case of model-based LES, whereas the Roe flux is used in the no-model simulation. For the sake of comparison, Table 1 compiles, together with the present DG results, a number of simulation results from the literature for the two Reynolds numbers considered, as well as the PIV experimental results of Parnaudeau et al. [5] at $R e=3900$. As regards the lower Re, we observe that overall the results from the no-model and the DG-VMS simulations are the closest to the experimental data. We know from previous studies [5] that the length of the recirculation bubble $L_{r}$ is one of 
Table 1 Simulation results for the flow past a circular cylinder at $R e=3900$ and 20000.

\begin{tabular}{|c|c|c|c|c|c|c|c|c|c|}
\hline \multicolumn{10}{|l|}{$R e=3900$} \\
\hline Author & Mach & Model & Mdofs & $T_{v s}$ & $C_{d}$ & $C l_{r m s}$ & St & $\frac{L_{r}}{D}$ & $-\frac{U_{\min }}{U_{c}}$ \\
\hline Parnaudeau et al.[5] & - & (Exp. PIV) & - & - & - & - & 0.208 & 1.51 & 0.34 \\
\hline Wornom et al.[7] & 0.1 & VMS-Wale & 1.80 & 30 & 0.99 & 0.11 & 0.210 & 1.45 & - \\
\hline Lysenko et al. [6] & 0.2 & TKE & 5.76 & 150 & 0.97 & 0.09 & 0.209 & 1.67 & 0.27 \\
\hline DG-P4 WALE & 0.2 & Wale & 2.59 & 100 & 0.96 & 0.09 & 0.209 & 1.69 & 0.31 \\
\hline DG-P4 VMS4 & 0.2 & VMS-Smag & 2.59 & 150 & 0.99 & 0.16 & 0.206 & 1.47 & 0.30 \\
\hline DG-P4 no-model & 0.2 & - & 2.59 & 150 & 1.00 & 0.15 & 0.209 & 1.42 & 0.30 \\
\hline \multicolumn{10}{|l|}{$R e=20000$} \\
\hline Wornom et al.[7] & 0.1 & VMS-Wale & 1.80 & 30 & 1.27 & 0.60 & 0.19 & 0.80 & - \\
\hline Lysenko et al. [8] & 0.2 & TKE & 5.76 & 75 & 1.39 & 0.73 & 0.17 & 0.59 & 0.18 \\
\hline Lysenko et al.[8] & 0.2 & TKE & 12.4 & 75 & 1.36 & 0.70 & 0.19 & 0.57 & 0.16 \\
\hline DG-P4 SMAG & 0.2 & Smag & 2.59 & 40 & 1.09 & 0.30 & 0.21 & 1.05 & 0.29 \\
\hline DG-P4 VMS2 & 0.2 & VMS-Smag. & 2.59 & 40 & 1.38 & 0.62 & 0.19 & 0.69 & 0.20 \\
\hline
\end{tabular}
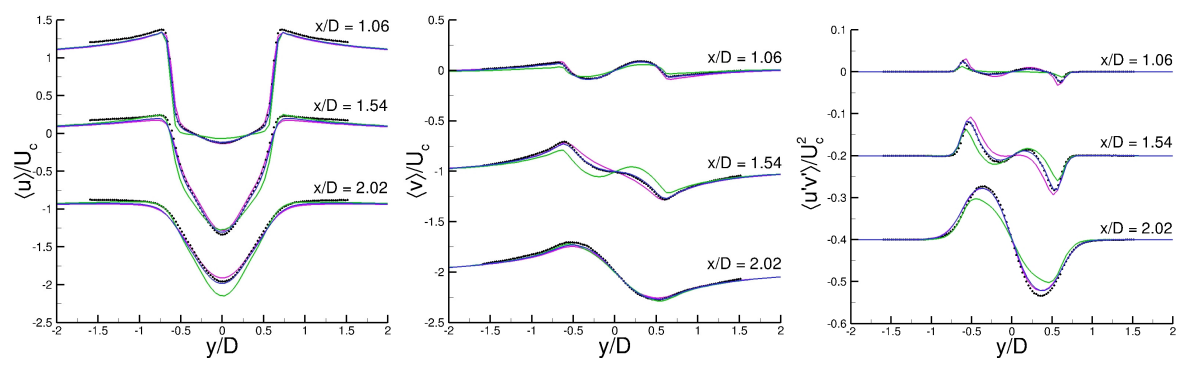

Fig. 3 Flow past a circular cylinder at $R e=3900$. Wake statistics at $x / D=1.06,1.54$, and 2.02. - : PIV [5], —: no-model DG, —: DG-VMS, —: DG-Wale.

the most sensitive quantities, as can be seen from the significant scatter in the values reported in Table 1. We see that the no-model DG approach leads to the lowest value of $L_{r} / D=1.42$, while the use of DG-Wale leads to the largest $L_{r} / D=1.69$, closer to the value of Lysenko et al. [6]. Finally, the value of $L_{r} / D=1.47$ from DG-VMS appears close to that of the experimental data and the values reported in [7]. Nevertheless, our values of root-mean-square of the lift coefficient $C_{l, r m s}$ are higher and those of the Strouhal number $S_{t}$ lower than those reported in [7], which might be interpreted as the result of a lower amount of numerical dissipation in the DG simulation. The values of the drag coefficient $C_{d}$ from the DG simulations are in line with those reported in the literature.

To examine in more detail the performance of the different approaches considered we have also compared the wake statistics with the reference PIV data reported in [5]. The outcome from this comparison can be seen in Fig. 3. A quick look at these figures, and in particular to the plot of $\left\langle u^{\prime} v^{\prime}\right\rangle / U_{c}^{2}$, shows that the DG-Wale exhibits the most significant discrepancies with the experimental data. Despite the fact that the no-model DG simulation clearly outperforms the standard LES approach, 
it is the DG-VMS simulation that yields the best agreement with the experimental data. These observations are corroborated by the results at the higher $R e=20000$. Indeed, despite the large scatter found in the data compiled in Table 1, it is fair to state that overall, the DG-VMS results are in line with the reference data from the literature, whereas the DG-Smag approach leads to an over-estimation of $L_{r}$ and $S t$.

\section{Conclusions}

The performance of the DG-VMS approach to LES of high-Reynolds-number turbulence has been assessed in two high-Reynolds-number configurations by detailed comparison with reference data from DNS and experiment. The DG-VMS results have also been contrasted to those from the no-model DG and the standard LES approaches. It appears from this research that the use of the DG-VMS approach in combination with the least dissipative possible numerical flux leads to the best match with the reference data for the two configurations considered. We have also highlighted the lack of robustness of the no-model DG approach for under-resolved simulations when the Reynolds number is high, as well as the over dissipative character of the standard approach to LES. Finally, a methodology has been proposed for the detailed evaluation of LES simulations on the TGV configuration in the context of very coarse discretisations.

Acknowledgements This research is partly funded by the European H2020 TILDA project. This work was performed using HPC resources from GENCI (Grants 2016-c20162a7622 and 2017A0022A10129). We thank F. Naddei for his help with generating the cylinder meshes.

\section{References}

1. Chapelier J.B., de la Llave Plata M., Lamballais E. (2016) Development of a multiscale LES model in the context of a modal DG method. Comput Meth Appl Mech Eng 307:275-299

2. de la Llave Plata M., Couaillier V., le Pape M.C. (2017) On the use of a highorder DG method for DNS and LES of wall-bounded turbulence. Comput Fluids, https://doi.org/10.1016/j.compfluid.2017.05.013

3. Dairay T., Lamballais E., Laizet S., Vassilicos J.C. (2017) Numerical dissipation vs. subgridscale modelling for LES. J Comput Phys 337:252-274

4. Moura R.C., Mengaldo G., Peiró J., Sherwin S.J.(2017) On the eddy-resolving capability of high-order DG approaches to implicit LES/under-resolved DNS of Euler turbulence. J Comput Phys 330:615-623

5. Parnaudeau P., Carlier J., Heitz D., Lamballais E. (2008) Experimental and numerical studies of the flow over a circular cylinder at Reynolds number 3900. Phys Fluids 20(8):085101

6. Lysenko D.A., Ertesvåg I.S., Rian K.E. (2012) LES of the flow over a circular cylinder at Reynolds number 3900 using the OpenFOAM toolbox. Flow Turbul Combust 89(4):491-518

7. Wornom S., Ouvrard H., Salvetti M.V. et al (2011) Variational multiscale LES of the flow past a circular cylinder: Reynolds number effects. Comput Fluids 47(1):44-50.

8. Lysenko D.A., Ertesvåg I.S., Rian K.E. (2014) LES of the flow over a circular cylinder at Reynolds number $2 \times 10^{4}$. Flow Turbul Combust 92 (2014):673-698 